\title{
10 Partial Differential Equations and Boundary Value Problems
}

\subsection{The Partial Differential Equations of Mathematical Physics}

Almost all of classical physics and a significant part of quantum physics involves only three types of partial differential equation (or PDE). These are

- Laplace's / Poisson's equation $\nabla^{2} \psi(r)=\sigma(r)$,

- the diffusion / heat conduction equation $D \nabla^{2} \psi(r, t)-\frac{\partial \psi}{\partial t}=\sigma(r, t)$, and

- the wave equation $\nabla^{2} \psi(r, t)-\frac{1}{c^{2}} \frac{\partial^{2} \psi}{\partial t^{2}}=\sigma(r, t)$.

In each case $\sigma$ represents a "source" or "sink" of the scalar field $\psi$; if it is zero, which happens in many applications, the equations become homogeneous. Familiar examples are provided by Maxwell's equations expressed in terms of the potentials $\boldsymbol{A}$ and $\Phi$. In SI units these reduce to the wave equation

$$
\nabla^{2} \boldsymbol{A}-\frac{1}{\mathrm{c}^{2}} \frac{\partial^{2} \boldsymbol{A}}{\partial \mathrm{t}^{2}}=-\mu_{0} \boldsymbol{j} \text { and } \nabla^{2} \Phi-\frac{1}{c^{2}} \frac{\partial^{2} \Phi}{\partial t^{2}}=-\frac{\rho}{\varepsilon_{0}}
$$

or, in the event of time independence, to Poisson's equation.

Pierre-Simon Laplace (1749-1827) was a French mathematician whose work was central to the development of both astronomy and statistics. Among his many accomplishments, he derived the equation and introduced the transform that bear his name. The Poisson equation (and distribution) are named after Simeon Denis Poisson (17811840) who was one of Laplace's students. Bonaparte made Laplace a count of the Empire in 1806. Demonstrating that he too could recognize genius, Louis-Phillipe made him a marquis in 1817, after the restoration of the monarchy.

What distinguishes the different physical phenomena that are described by any one of these equations are the identity of the scalar field $\psi$ (i.e. whether it is an electrostatic potential, a temperature, a density, a transverse displacement of a vibrating medium, or what have you) and the boundary conditions and, where time is an independent variable, the initial conditions that are imposed on it. Indeed, it is only when a partial differential equation is accompanied by such conditions that it will admit a unique solution.

The impact of boundary conditions or more precisely of the geometrical character of the boundaries is first experienced in the choice of coordinate system to use when $\psi$ is defined on a multi-dimensional space. It is enormously convenient to be able to specify the boundary of the domain or region of definition by means of fixed values of one or more of the coordinates. Thus, for example, if the boundary is a rectangular box, which can be specified by $x=a$ and $b, y=c$ and $d, z=e$ and $f$, where $a, b, c, d, e$ and $f$ are constants, one should choose Cartesian coordinates; if it is a sphere, which can be specified by $r=a$ constant, $0 \leqslant \theta \leqslant \pi, 0 \leqslant \varphi \leqslant 2 \pi$, use spherical polars; 
and if it is a cylinder, corresponding to $\rho=R, z=a$ and $b, 0 \leqslant \varphi \leqslant 2 \pi$, where $R, a$ and $b$ are constants, cylindrical polars are the obvious choice. Consequently, we need to be able to express the $\nabla^{2}$ differential operator in any type of curvilinear coordinate system.

\subsection{Curvilinear Coordinates}

A point in space can be described by any three independent parameters $\left(u_{1}, u_{2}, u_{3}\right)$. To move in either direction between such a system of coordinates and the Cartesian system, there must be some definite functional relationship relating the two sets of coordinates at any point:

$$
x=f_{1}\left(u_{1}, u_{2}, u_{3}\right), \quad y=f_{2}\left(u_{1}, u_{2}, u_{3}\right), \quad z=f_{3}\left(u_{1}, u_{2}, u_{3}\right)
$$

and

$$
u_{1}=F_{1}(x, y, z), \quad u_{2}=F_{2}(x, y, z), \quad u_{3}=F_{3}(x, y, z) .
$$

The three coordinate surfaces $u_{i}=a$ constant can be drawn. If the orientations of these surfaces change from point to point, the $u_{i}$ are called curvilinear coordinates and if the three surfaces are mutually perpendicular everywhere, they are called orthogonal curvilinear coordinates.

At any point, specified by the radius vector from the origin $\boldsymbol{r}=x \boldsymbol{i}+y \boldsymbol{j}+z \boldsymbol{k}$, we can construct unit vectors $\boldsymbol{e}_{i}$ normal to the surfaces $u_{i}=a$ constant by means of

$$
\boldsymbol{e}_{i} \equiv \frac{\partial \boldsymbol{r} / \partial u_{i}}{\left|\partial \boldsymbol{r} / \partial u_{i}\right|}
$$

These clearly form an orthogonal system when the coordinates are orthogonal. The quantities

$$
h_{i}=\left|\partial \boldsymbol{r} / \partial u_{i}\right|=\sqrt{\left(\frac{\partial x}{\partial u_{i}}\right)^{2}+\left(\frac{\partial y}{\partial u_{i}}\right)^{2}+\left(\frac{\partial z}{\partial u_{i}}\right)^{2}}
$$

are called scale factors and depend upon the position of $\boldsymbol{r}$ in space.

Consider a small displacement $d \boldsymbol{r}=d x \boldsymbol{i}+d y \boldsymbol{j}+d z \boldsymbol{k}$. Its curvilinear components can be read off from

$$
d \boldsymbol{r}=\frac{\partial \boldsymbol{r}}{\partial u_{1}} d u_{i}+\frac{\partial \boldsymbol{r}}{\partial u_{2}} d u_{2}+\frac{\partial \boldsymbol{r}}{\partial u_{3}} d u_{3}=h_{1} d u_{i} \boldsymbol{e}_{1}+h_{2} d u_{2} \boldsymbol{e}_{2}+h_{3} d u_{3} \boldsymbol{e}_{3} .
$$

Let us assume that the curvilinear system is orthogonal. The line element or element of arc length is then given by the square root of

$$
d s^{2}=d \boldsymbol{r} \cdot d \boldsymbol{r}=h_{1}^{2}\left(d u_{1}\right)^{2}+h_{2}^{2}\left(d u_{2}\right)^{2}+h_{3}^{2}\left(d u_{3}\right)^{2} .
$$


In addition, the volume element, the volume of the parallelepiped formed by the surfaces

$$
u_{1}=c_{1}, u_{1}=c_{1}+d u_{1}, u_{2}=c_{2}, u_{2}=c_{2}+d u_{2}, u_{3}=c_{3}, u_{3}=c_{3}+d u_{3},
$$

is

$$
d V=h_{1} h_{2} h_{3} d u_{1} d u_{2} d u_{3}
$$

To express grad $\psi$ in terms of curvilinear coordinates we start with

$$
(\operatorname{grad} \psi) \cdot d \boldsymbol{r}=d \psi \equiv \frac{\partial \psi}{\partial u_{1}} d u_{1}+\frac{\partial \psi}{\partial u_{2}} d u_{2}+\frac{\partial \psi}{\partial u_{3}} d u_{3}
$$

and rewrite it in the form

$$
(\operatorname{grad} \psi) \cdot d \boldsymbol{r}=\left(\frac{1}{h_{1}} \frac{\partial \psi}{\partial u_{1}}\right) h_{1} d u_{1}+\left(\frac{1}{h_{2}} \frac{\partial \psi}{\partial u_{2}}\right) h_{2} d u_{2}+\left(\frac{1}{h_{3}} \frac{\partial \psi}{\partial u_{3}}\right) h_{3} d u_{3} .
$$

It follows immediately that

$$
\operatorname{grad} \psi=\frac{1}{h_{1}} \frac{\partial \psi}{\partial u_{1}} \boldsymbol{e}_{1}+\frac{1}{h_{2}} \frac{\partial \psi}{\partial u_{2}} \boldsymbol{e}_{2}+\frac{1}{h_{3}} \frac{\partial \psi}{\partial u_{3}} \boldsymbol{e}_{3} .
$$

To determine the divergence of a vector field $\boldsymbol{A}$ we make use of the definition

$$
\boldsymbol{\nabla} \cdot \boldsymbol{A} \equiv \lim _{\Delta V \rightarrow 0} \frac{\int_{\Delta S} \boldsymbol{A} \cdot d s}{\Delta V}=\lim _{\Delta V \rightarrow 0} \frac{\text { net flux of } \boldsymbol{A} \text { through surface } \Delta \mathrm{S} \text { bounding } \Delta V}{\Delta V}
$$

and note that the flux through an elementary area oriented perpendicular to the $\boldsymbol{e}_{1} \mathrm{di}$ rection is $A_{1} h_{2} d u_{2} h_{3} d u_{3}$. Thus, the net flux of $\boldsymbol{A}$ through two such areas separated by a distance $h_{1} d u_{1}$ is

$$
h_{1} d u_{1} \frac{1}{h_{1}} \frac{\partial}{\partial u_{1}}\left(A_{1} h_{2} h_{3}\right) d u_{2} d u_{3}
$$

Adding the corresponding contributions from the other four faces of a volume element and dividing by its volume, we obtain

$$
\operatorname{div} \boldsymbol{A}=\frac{1}{h_{1} h_{2} h_{3}}\left[\frac{\partial}{\partial u_{1}}\left(A_{1} h_{2} h_{3}\right)+\frac{\partial}{\partial u_{2}}\left(A_{2} h_{3} h_{1}\right)+\frac{\partial}{\partial u_{3}}\left(A_{3} h_{1} h_{2}\right)\right] .
$$

The expression for the Laplacian is obtained by combining the formulas for gradient and divergence:

$$
\nabla^{2} \psi=\frac{1}{h_{1} h_{2} h_{3}}\left[\frac{\partial}{\partial u_{1}}\left(\frac{h_{2} h_{3}}{h_{1}} \frac{\partial \psi}{\partial u_{1}}\right)+\frac{\partial}{\partial u_{2}}\left(\frac{h_{3} h_{1}}{h_{2}} \frac{\partial \psi}{\partial u_{2}}\right)+\frac{\partial}{\partial u_{3}}\left(\frac{h_{1} h_{2}}{h_{3}}\right)\right] .
$$

Examples: The most common curvilinear coordinates in physics are

- plane polar: $x=r \cos \theta, \quad y=r \sin \theta$ 
- cylindrical polar: $x=r \cos \theta, \quad y=r \sin \theta, z=z$

- $\quad$ spherical polar: $x=r \sin \theta \cos \varphi, \quad y=r \sin \theta \sin \varphi, \quad z=r \cos \theta$.

The scale factors $h_{i}$ are defined for any set of coordinates by

$$
h_{i}=\sqrt{\left(\frac{\partial x}{\partial u_{i}}\right)^{2}+\left(\frac{\partial y}{\partial u_{i}}\right)^{2}+\left(\frac{\partial z}{\partial u_{i}}\right)^{2}} .
$$

Thus, for spherical polars, we have

$$
h_{r}=1, \quad h_{\theta}=r, \quad h_{\varphi}=r \sin \theta
$$

and so,

$$
\begin{gathered}
d s^{2}=d r^{2}+r^{2} d \theta^{2}+r^{2} \sin ^{2} \theta d \varphi^{2}, \\
d V=r^{2} \sin \theta d r d \theta d \varphi \\
\operatorname{div} \boldsymbol{A}=\frac{1}{r^{2} \sin \theta}\left[\frac{\partial}{\partial r}\left(r^{2} \sin \theta A_{r}\right)+\frac{\partial}{\partial \theta}\left(r \sin \theta A_{\theta}\right)+\frac{\partial}{\partial \varphi}\left(r A_{\varphi}\right)\right]
\end{gathered}
$$

or,

$$
\operatorname{div} \boldsymbol{A}=\frac{\partial A_{r}}{\partial r}+\frac{2}{r} A_{r}+\frac{1}{r} \frac{\partial A_{\theta}}{\partial \theta}+\frac{\cot \theta}{r} A_{\theta}+\frac{1}{r \sin \theta} \frac{\partial A_{\varphi}}{\partial \varphi}
$$

and,

$$
\nabla^{2} \psi=\frac{1}{r^{2}} \frac{\partial}{\partial r}\left(r^{2} \frac{\partial \psi}{\partial r}\right)+\frac{1}{r^{2} \sin \theta} \frac{\partial}{\partial \theta}\left(\sin \theta \frac{\partial \psi}{\partial \theta}\right)+\frac{1}{r^{2} \sin ^{2} \theta} \frac{\partial^{2} \psi}{\partial \varphi^{2}} .
$$

An analogous determination in cylindrical polars can be easily done and one finds in particular that the Laplacian is

$$
\nabla^{2} \psi=\frac{\partial^{2} \psi}{\partial r^{2}}+\frac{1}{r} \frac{\partial \psi}{\partial r}+\frac{1}{r^{2}} \frac{\partial^{2} \psi}{\partial \theta^{2}}+\frac{\partial^{2} \psi}{\partial z^{2}}
$$

\subsection{Separation of Variables}

Various methods have been devised for the solution of partial differential equations corresponding to different kinds of boundaries (whether finite or at infinity) and different kinds of boundary and initial conditions. What we shall come to recognize is that they all involve representation of the solution as an expansion in terms of eigenfunctions of one or more of the partial differential operators in the equation which achieves a separation of the dependence on the individual coordinate variables involved. Eigenfunction is a term that we have not encountered before. Therefore, to provide some context, we shall present a method of solution that is actually called the separation of variables method. We will then abstract from it the key elements that are common to all methods of solution. 
The "laboratory" we will use to investigate separation of variables is the vibrating string problem. Suppose that we have a string of mass per unit length $\rho$ stretched under a tension T along the line $x=0$ to $x=L$ and fixed at both ends. The string is set in motion at time $t=0$ by means of some combination of plucking and striking it. Denoting the transverse displacement of the string by $\psi(x, t)$, the string's equation of motion (obtained by an application of Newton's $2^{\text {nd }}$ law) is the one-dimensional wave equation

$$
\frac{\partial^{2} \psi}{\partial x^{2}}=\frac{1}{c^{2}} \frac{\partial^{2} \psi}{\partial t^{2}} \text { where } c=\sqrt{\frac{T}{\rho}}
$$

The manner in which motion is initiated is described by the initial conditions $\psi(x, 0)=u_{0}(x)$ and $\left.\frac{\partial \psi(x, t)}{\partial t}\right|_{t=0}=v_{0}(x)$, where $u_{0}(x)$ and $v_{0}(x)$ are known functions. Finally, the fact that the string has fixed end-points is captured by the boundary conditions $\psi(0, t)=0$ and $\psi(L, t)=0$.

One can prove that the solution of a linear partial differential equation accompanied by a complete set of boundary/initial conditions is unique. Thus, if we find a solution, no matter by what means, we are assured that it is the only solution to the problem. The means we shall employ here begins with the assumption that the motion at any point $0 \leqslant x \leqslant L$, and time $t \geqslant 0$, can be expressed in the form

$$
\psi(x, t)=X(x) T(t) .
$$

If our method works, if we obtain a solution, this assumption will be justified a posteriori.

Substituting into the wave equation and dividing through by $\psi=X T$ we find

$$
\frac{1}{X(x)} \frac{d^{2} X}{d x^{2}}=-\lambda=\frac{1}{c^{2}} \frac{1}{T(t)} \frac{d^{2} T}{d t^{2}},
$$

where $\lambda$ must be a constant since the first equality implies it is independent of $t$ while the second equality implies it is independent of $x$. The two equalities yield the same ordinary differential equation (ODE) with constant coefficients. The general solutions are

$$
X_{\lambda}(x)=\left\{\begin{array}{cc}
A \cos \sqrt{\lambda} x+B \sin \sqrt{\lambda} x & \text { if } \lambda>0 \\
A e^{\sqrt{-\lambda} x}+B e^{-\sqrt{-\lambda} x} & \text { if } \lambda<0 \\
A x+B & \text { if } \lambda=0
\end{array}\right.
$$

and

$$
T_{\lambda}(t)=\left\{\begin{array}{cl}
A \cos \sqrt{\lambda} c t+B \sin \sqrt{\lambda} c t & \text { if } \lambda>0 \\
A e^{\sqrt{-\lambda} c t}+B e^{-\sqrt{-\lambda} c t} & \text { if } \lambda<0 . \\
A c t+B & \text { if } \lambda=0
\end{array}\right.
$$

The initial conditions on $\psi(x, t)$ involve functions of $x$ and so place no restrictions on $T_{\lambda}(t)$ other than a general requirement of boundedness on $0 \leqslant t<\infty$. Such conditions are called non-homogeneous. In contrast, the boundary conditions on $\psi(x, t)$ 
are homogeneous: they require that $\psi(x, t)$ and hence each $X_{\lambda}(x)$ vanish at $x=0$ and $x=L$. A quick check tells us that these conditions cannot be satisfied for any values of $\lambda \leqslant 0$ (except in the trivial case of $A=B=0$ ). On the other hand, there is an infinite set of values of $\lambda>0, \lambda_{n}=\frac{n^{2} \pi^{2}}{L^{2}}, n=1,2,3, \ldots$, which admit these conditions and the corresponding solutions are, to within an arbitrary multiplicative constant, $X_{n}(x)=\sin \frac{n \pi x}{L}$. The latter are called the eigenfunctions of the differential operator $\frac{d^{2}}{d x^{2}}$ appropriate to these boundary conditions and the $\lambda_{n}$ are its eigenvalues (characteristic values). This means that of those functions that vanish at $x=0$ and $x=L$, there is a unique subset, $\left\{X_{n}\right\}$, with the property that when operated on by $\frac{d^{2}}{d x^{2}}$ they are reproduced multiplied by a characteristic constant (an eigenvalue).

With $\lambda$ determined, so is $T_{\lambda}$. In fact, we now have an infinite set of factored solutions

$$
\psi_{n}(x, t)=X_{n}(x) T_{n}(t)=\sin \frac{n \pi x}{L}\left(A_{n} \cos \frac{n \pi c t}{L}+B_{n} \sin \frac{n \pi c t}{L}\right), \quad n=1,2,3, \ldots,
$$

each of which satisfies both the wave equation and the boundary conditions. Moreover, because the equation is linear, every linear combination of solutions satisfies both the wave equation and the boundary conditions. But, what about the initial conditions? Evidently, unless $u_{0}(x)$ and $v_{0}(x)$ are themselves sinusoidal with period $2 L$, we will not be able to reproduce them with one or even a linear combination of several of the $\psi_{n}(x, t)$. Therefore, we shall use all of them. We form the superposition

$$
\psi(x, t)=\sum_{n=1}^{\infty} \sin \frac{n \pi x}{L}\left(A_{n} \cos \frac{n \pi c t}{L}+B_{n} \sin \frac{n \pi c t}{L}\right)
$$

and impose the initial conditions via

$$
\begin{aligned}
& u_{0}(x)=\sum_{n=1}^{\infty} \sin \frac{n \pi x}{L} A_{n}, \text { and } \\
& v_{0}(x)=\sum_{n=1}^{\infty} \sin \frac{n \pi x}{L} B_{n} \frac{n \pi c}{L} .
\end{aligned}
$$

We recognize the summations in these three equations as Fourier sine series. Thus, so long as $u(x, t), u_{0}(x)$ and $v_{0}(x)$ are continuous functions of $x$, the series will converge uniformly to these functions when $A_{n}$ and $\frac{n \pi c}{L} B_{n}$ are replaced by the Fourier sine coefficients of $u_{0}(x)$ and $v_{0}(x)$, respectively; that is, when

$$
\begin{gathered}
A_{n}=\frac{2}{L} \int_{0}^{L} u_{0}(x) \sin \frac{n \pi x}{L} d x, \text { and } \\
B_{n}=\frac{2}{n \pi c} \int_{0}^{L} v_{0}(x) \sin \frac{n \pi x}{L} d x .
\end{gathered}
$$


This completes the solution of the problem.

Evidently, the method of separation of variables works. Let us be certain we understand why.

Working backwards, we see that a critical element is the implicit representation of $\psi(x, t)$ by the eigenfunction expansion

$$
\psi(x, t)=\sum_{n=1}^{\infty} b_{n}(t) \sin \frac{n \pi x}{L}
$$

which is a Fourier sine series, every term of which satisfies the boundary conditions. Thus, it must converge uniformly to $\psi(x, t)$ and so, when we substitute it into the wave equation, we can interchange the order of summation and integration. The result is

$$
\sum_{n=1}^{\infty} b_{n}(t) \frac{d^{2}}{d x^{2}} \sin \frac{n \pi x}{L}=\sum_{n=1}^{\infty} b_{n}(t)\left(\frac{-n^{2} \pi^{2}}{L^{2}}\right) \sin \frac{n \pi x}{L}=\sum_{n=1}^{\infty} \frac{1}{c^{2}} \frac{d^{2} b_{n}(t)}{d t^{2}} \sin \frac{n \pi x}{L} .
$$

Because the sine functions are orthogonal, the second equality implies that

$$
\frac{d^{2} b_{n}}{d t^{2}}+\left(\frac{n \pi c}{L}\right)^{2} b_{n}(t)=0
$$

and hence, that

$$
b_{n}(t)=A_{n} \cos \frac{n \pi c t}{L}+B_{n} \sin \frac{n \pi c t}{L} .
$$

The solution is then completed by relating $A_{n}$ and $B_{n}$ to the Fourier sine coefficients of $u_{0}(x)$ and $v_{0}(x)$.

The separation of variables method is successful because it amounts to an expansion of $\psi(x, t)$ in terms of the eigenfunctions of the differential operator associated with homogeneous boundary conditions. The differential operator is replaced by its eigenvalues and thereby eliminated from the partial differential equation. The PDE is replaced by a series of ODE's with constant coefficients.

That being said, we shall now perform a practical inventory of the steps that comprise this method and do so in the course of solving another boundary value problem. The problem is to find the electrostatic potential everywhere inside a conducting rectangular box of dimensions $a \times b \times c$ which has all of its walls grounded except for the top which is separated from the other walls by thin insulating strips and maintained at a potential $V$.

The PDE to be solved is Laplace's equation

$$
\nabla^{2} \psi=0
$$

Here is how we proceed with its solution.

Step 1. Choose an appropriate coordinate system.

We choose Cartesian coordinates with the origin at one corner of the box so that its interior and boundaries are defined by $0 \leqslant x \leqslant a, 0 \leqslant y \leqslant b, 0 \leqslant z \leqslant c$. The boundary conditions then become

$$
\psi(0, y, z)=\psi(a, y, z)=\psi(x, 0, z)=\psi(x, b, z)=\psi(x, y, 0)=0 \text { and } \psi(x, y, c)=V .
$$


Step 2. Separate the PDE into ODE's.

Substitute $\psi(x, y, z)=X(x) Y(y) Z(z)$ into the PDE and then divide through by $\psi$ to obtain

$$
\frac{1}{X} \frac{d^{2} X}{d x^{2}}+\frac{1}{Y} \frac{d^{2} Y}{d^{2} y}+\frac{1}{Z} \frac{d^{2} Z}{d z^{2}}=0
$$

This can hold for all $x, y$ and $z$ if and only if each term is separately equal to a constant with the three constants summing to zero:

$$
\frac{d^{2} X}{d x^{2}}=\lambda_{1} X, \frac{d^{2} Y}{d y^{2}}=\lambda_{2} Y \text { and } \frac{d^{2} Z}{d z^{2}}=\lambda_{3} Z \text { with } \lambda_{1}+\lambda_{2}+\lambda_{3}=0 .
$$

These three DE's are identical to each other and to the separated DE's of the stretched string problem. Therefore, they have the same three sets of solutions (10.3.4) corresponding to positive, negative and null values of the separation constants.

Step 3. Impose the single-coordinate boundary conditions that are homogeneous at both boundaries and solve the corresponding eigenvalue equations for the functions of those coordinates.

In this case, the homogeneous boundary conditions require that

$$
X(0)=X(a)=0, Y(0)=Y(b)=0 .
$$

We know from the stretched string problem that this implies eigenvalues

$$
\lambda_{1}=-\left(\frac{n \pi}{a}\right)^{2}, n=1,2, \ldots \text { and } \lambda_{2}=-\left(\frac{m \pi}{b}\right)^{2}, m=1,2, \ldots,
$$

corresponding to the eigenfunctions

$$
X_{n}(x)=\sin \frac{n \pi x}{a} \text { and } Y_{m}(y)=\sin \frac{m \pi y}{b} .
$$

Notice that while we know that $Z(0)=0$ we have no information bearing directly on $Z(c)$.

Step 4. Solve for the remaining function(s).

We now know that

$$
\lambda_{3}=\left(\frac{n \pi}{a}\right)^{2}+\left(\frac{m \pi}{b}\right)^{2} .
$$

Since this is always positive, the corresponding solution for $Z(z)$ is a linear combination of $e^{\sqrt{\lambda_{3}} z}$ and $e^{-\sqrt{\lambda_{3}} z}$. But we must also satisfy $Z(0)=0$. Therefore, an appropriate linear combination is

$$
Z_{n m}(z)=\sinh \left(\sqrt{\left(\frac{n \pi}{a}\right)^{2}+\left(\frac{m \pi}{b}\right)^{2}} z\right) .
$$

Step 5. Form a linear superposition of all factored solutions.

We now have a doubly infinite set of factored solutions

$$
X_{n}(x) Y_{m}(y) Z_{n m}(z), \quad n, m=1,2, \ldots
$$


each of which satisfies the PDE as well as the homogeneous boundary conditions. To prepare for the imposition of the non-homogeneous boundary condition, we take advantage of the linearity of the PDE and form the superposition

$$
\psi(x, y, z)=\sum_{n=1}^{\infty} \sum_{m=1}^{\infty} A_{n m} \sin \frac{n \pi x}{a} \sin \frac{m \pi y}{b} \sinh \left(\sqrt{\left(\frac{n \pi}{a}\right)^{2}+\left(\frac{m \pi}{b}\right)^{2}} z\right) .
$$

Step 6. Impose the remaining boundary condition(s).

In this case there remains only one condition: $\psi(x, y, c)=V$. Imposing it on our superposition we have the requirement that

$$
V=\sum_{n=1}^{\infty} \sum_{m=1}^{\infty} A_{n m} \sin \frac{n \pi x}{a} \sin \frac{m \pi y}{b} \sinh \left(\sqrt{\left(\frac{n \pi}{a}\right)^{2}+\left(\frac{m \pi}{b}\right)^{2}} c\right) .
$$

This is a double Fourier sine series and so we can use the Euler formula for the coefficients of such series to determine $A_{n m}$. Thus,

$$
A_{n m}=\frac{V}{\sinh \left(\sqrt{\left(\frac{n \pi}{a}\right)^{2}+\left(\frac{m \pi}{b}\right)^{2} c}\right)} \frac{4}{a b} \int_{0}^{a} \int_{0}^{b} \sin \frac{n \pi x}{a} \sin \frac{m \pi y}{b} d x d y
$$

or,

$$
A_{n m}=\frac{V}{\sinh \left(\sqrt{\left(\frac{n \pi}{a}\right)^{2}+\left(\frac{m \pi}{b}\right)^{2} c}\right)} \frac{4}{n m \pi^{2}}\left(1-(-1)^{n}\right)\left(1-(-1)^{m}\right) .
$$

Substituting back into the superposition we obtain as our solution

$$
\psi(x, y, z)=\frac{16 V}{\pi^{2}} \sum_{n=1,3,5, \ldots}^{\infty} \sum_{m=1,3,5, \ldots}^{\infty} \frac{1}{n m} \sin \frac{n \pi x}{a} \sin \frac{m \pi y}{b} \frac{\sinh \left(\sqrt{\left(\frac{n \pi}{a}\right)^{2}+\left(\frac{m \pi}{b}\right)^{2} z}\right)}{\sinh \left(\sqrt{\left(\frac{n \pi}{a}\right)^{2}+\left(\frac{m \pi}{b}\right)^{2} c}\right)} .
$$

\subsection{What a Difference the Choice of Coordinate System Makes!}

We shall now investigate the solution of the homogeneous versions of the partial differential equations we introduced in Section 10.1 when applied to a three dimensional medium with either rectangular, spherical or cylindrical symmetry.

Recall that the PDE's are

$$
\begin{aligned}
\nabla^{2} \psi & =0, \\
\nabla^{2} \psi-\frac{1}{D} \frac{\partial \psi}{\partial t} & =0, \\
\nabla^{2} \psi-\frac{1}{c^{2}} \frac{\partial^{2} \psi}{\partial t^{2}} & =0
\end{aligned}
$$


We start by separating off the time dependence in the case of the last two of these. We do this by assuming a solution of the form

$$
\psi(\boldsymbol{r}, t)=u(\boldsymbol{r}) T(t) .
$$

Substituting into the diffusion equation and dividing by $\psi=u T$ we find

$$
\frac{1}{u(\boldsymbol{r})} \nabla^{2} u(\boldsymbol{r})=-\lambda=\frac{1}{D} \frac{1}{T(t)} \frac{d T}{d t},
$$

where $\lambda$ must be a constant since the first equality implies it is independent of $t$ and the second equality implies it is independent of $\boldsymbol{r}$. The second equality is a simple differential equation for $T(t)$ with solution

$$
T(t)=A e^{-\lambda D t} .
$$

Unlike the vibrating string problem, we will not assume an initial condition but require only that $T(t)$ be bounded for $0 \leqslant t<\infty$, (i.e. that it satisfy the homogeneous boundary condition $|T(\infty)|<\infty)$. This means that $\lambda$ must be positive and so we set $\lambda=k^{2}$. Thus,

$$
T(t)=A e^{-k^{2} D t}
$$

and the first part of (10.4.3) is

$$
\nabla^{2} u(\boldsymbol{r})+k^{2} u(\boldsymbol{r})=0
$$

which is called Helmholtz' equation. Note that Laplace's equation is the special case of Helmholtz' equation corresponding to $k^{2}=0$.

The Helmholtz' equation is named for Hermann von Helmholtz (1821-1894), a German physician and physicist. He made significant contributions in neurophysiology, physics (electrodynamics and thermodynamics), and philosophy. The Helmholtz Association of German research centres is named after him.

We can do a similar separation of the time dependence in the case of the wave equation. Assuming a solution of the form (10.4.2), substituting into the equation, and dividing by $\psi=u T$, we find

$$
\frac{1}{u} \nabla^{2} u=-\lambda=\frac{1}{c^{2}} \frac{1}{T} \frac{d^{2} T}{d t^{2}}
$$

with $\lambda$ a constant. The second part of (10.4.6) is the same differential equation for $T$ that we encountered in the vibrating string problem. This time we will accompany it with the homogeneous boundary condition $|T( \pm \infty)|<\infty$ which makes it an eigenvalue equation with solution $\lambda=k^{2}, 0 \leqslant k<\infty$ and

$$
T_{\lambda}(t)=T_{k}(t)=A_{k} e^{i k c t}+B_{k} e^{-i k c t}=\left\{\begin{array}{c}
e^{i k c t} \\
e^{-i k c t}
\end{array}\right\}=\left\{\begin{array}{c}
\sin k c t \\
\cos k c t
\end{array}\right\} .
$$


The use of curly braces in (10.4.7) is a convenient short-hand for a linear combination of the functions that appear between them.

This means that we obtain the Helmholtz equation

$$
\nabla^{2} u+k^{2} u=0
$$

once again to describe the space dependence.

To proceed further with the separation of variables we must now adopt one or another type of coordinate system.

In Cartesian coordinates Helmholtz' equation is

$$
\frac{\partial^{2} u}{\partial x^{2}}+\frac{\partial^{2} u}{\partial y^{2}}+\frac{\partial^{2} u}{\partial z^{2}}+k^{2} u=0
$$

and so, assuming a separated solution of the form $u=X(x) Y(y) Z(z)$, substituting into (10.4.8), and dividing through by $u=X Y Z$, we have

$$
\frac{1}{X} \frac{d^{2} X}{d x^{2}}+\frac{1}{Y} \frac{d^{2} Y}{d y^{2}}+\frac{1}{Z} \frac{d^{2} Z}{d z^{2}}+k^{2}=0
$$

Thus,

$$
\frac{1}{X} \frac{d^{2} X}{d x^{2}}=-\lambda_{1}=-k^{2}-\frac{1}{Y} \frac{d^{2} Y}{d y^{2}}-\frac{1}{Z} \frac{d^{2} Z}{d z^{2}},
$$

where $\lambda_{1}$ must be a (separation) constant. Then, as in the potential problem of the last Section, we find

$$
\frac{1}{Y} \frac{d^{2} Y}{d y^{2}}=-\lambda_{2}
$$

and,

$$
\frac{1}{Z} \frac{d^{2} Z}{d z^{2}}=-\lambda_{3}
$$

but now

$$
\lambda_{1}+\lambda_{2}+\lambda_{3}=k^{2}
$$

We now need some information about the spatial boundaries. Rather than confine the medium to a finite box as was the case in the potential problem, we shall assume that the medium is infinite and that the boundary conditions require $X, Y$, and $Z$ to be bounded for all $x, y$, and $z$. The differential equations for $X, Y$, and $Z$ are the same as the differential equation for $T(t)$ and so we know that they admit bounded solutions if and only if $\lambda_{i} \geqslant 0$ for $i=1,2$, and 3. Thus, we set $\lambda_{1}=k_{1}^{2}, \lambda_{2}=k_{2}^{2}, \lambda_{3}=k_{3}^{2}$ with $-\infty<k_{1}, k_{2}, k_{3}<\infty$ to obtain

$$
X(x) \propto e^{i k_{1} x}
$$




$$
\begin{gathered}
Y(y) \propto e^{i k_{2} y} \\
Z(z) \propto e^{i k_{3} z},
\end{gathered}
$$

with

$$
k_{1}^{2}+k_{2}^{2}+k_{3}^{2}=\boldsymbol{k}^{2}
$$

Multiplying these together we get (plane wave) solutions for $u(r)$ of the form

$$
u(\boldsymbol{r})=A_{k} e^{i \boldsymbol{k} \cdot \boldsymbol{r}}
$$

where $\boldsymbol{k}$ is a three-dimensional vector with norm $\boldsymbol{k} \cdot \boldsymbol{k}=k^{2}$.

This is as far as we can go without having information about how the wave motion or diffusion was initiated, that is, without having initial conditions to impose. Therefore, let us turn instead to the question of the kind of waves we would obtain if there was cylindrical geometry.

In cylindrical coordinates Helmholtz' equation is

$$
\frac{\partial^{2} u}{\partial r^{2}}+\frac{1}{r} \frac{\partial u}{\partial r}+\frac{1}{r^{2}} \frac{\partial^{2} u}{\partial \theta^{2}}+\frac{\partial^{2} u}{\partial z^{2}}+k^{2} u=0
$$

Assuming a separated solution of the form $u=R(r) \Theta(\theta) Z(z)$, substituting in (10.4.17) , and dividing through by $u=R \Theta Z$, we find

$$
\frac{1}{R}\left[\frac{d^{2} R}{d r^{2}}+\frac{1}{r} \frac{d R}{d r}\right]+\frac{1}{r^{2}} \frac{1}{\Theta} \frac{d^{2} \Theta}{d \theta^{2}}+\frac{1}{Z} \frac{d^{2} Z}{d z^{2}}+k^{2}=0 .
$$

Separating variables yields the equations

$$
\begin{gathered}
\frac{1}{Z} \frac{d^{2} Z}{d z^{2}}=-\lambda_{2} \\
\frac{1}{\Theta} \frac{d^{2} \Theta}{d \theta^{2}}=-\lambda_{1} \\
\frac{1}{R}\left[\frac{d^{2} R}{d r^{2}}+\frac{1}{r} \frac{d R}{d r}\right]-\frac{\lambda_{1}}{r^{2}}-\lambda_{2}+k^{2}=0 .
\end{gathered}
$$

Most physical applications involve the boundary condition $\Theta(\theta+2 \pi)=\Theta(\theta)$ to ensure that $\Theta$ is a single valued function. It then follows that $\lambda_{1}=m^{2}, m=0,1,2, \ldots$ and

$$
\Theta(\theta) \equiv \Theta_{m}(\theta)=A_{m} \cos m \theta+B_{m} \sin m \theta
$$

are the eigensolutions (or characteristic solutions) of equation (10.4.19) .

There is no common boundary condition that can be applied to the solutions of (10.4.20) and so we write them for now as

$$
Z(z)=C \exp \left(\sqrt{-\lambda_{2}} z\right)+D \exp \left(-\sqrt{-\lambda_{2}} z\right)
$$


and note that this will be a trigonometric function if $\lambda_{2}>0$ and a hyperbolic function if $\lambda_{2}<0$.

Setting $k^{2}-\lambda_{2}=\alpha^{2}$ and $\rho=\alpha r$, equation (10.4.21) becomes

$$
\frac{d^{2} R}{d \rho^{2}}+\frac{1}{\rho} \frac{d R}{d \rho}+\left(1-\frac{m^{2}}{\rho^{2}}\right) R=0
$$

which is Bessel's equation. As we have seen, its general solution can be expressed as the linear combination

$$
R(r)=E J_{m}(\alpha r)+F N_{m}(\alpha r)
$$

where $J_{m}(x)$ and $N_{m}(x)$ are the Bessel and Neumann functions of order $m$, respectively. These have an oscillatory dependence on $x$ with an infinite number of zeros. Moreover, the Neumann function, $N_{m}(x)$, is singular at $x=0$. Thus, if there are homogeneous boundary conditions such as $R(0)=R(a)=0$, we would require $F=0$ for all $m$ and determine eigensolutions $J_{m}\left(\alpha_{m, n} r\right)$ where $\alpha_{m, n}=x_{m, n} / a$ and $x_{m, n}$ is the nth zero of $J_{m}(x)$.

If $\lambda_{2}>k^{2}, \alpha$ will be a pure imaginary. In that case it is conventional to replace (10.4.25) by the linear combination

$$
R(r)=G_{I m}(|\alpha| r)+H_{K_{m}}(|\alpha| r)
$$

where $I_{m}(x)$ and $K_{m}(x)$ are called modified Bessel functions.The modified Bessel functions are not oscillatory in behaviour but rather behave exponentially for large $x$. Specifically, $I_{m} \rightarrow \infty$ and $K_{m} \rightarrow 0$ as $x \rightarrow \infty$. At the other end of the scale, as $x \rightarrow 0, K_{m} \rightarrow \infty$ while $I_{m} \rightarrow 0$ if $m \neq 0$ and $I_{0} \rightarrow 1$. Note that the modified Bessel functions arise when the $\mathrm{z}$ - dependence is given by oscillatory sine and cosine functions. The converse is true also: if $Z(z)$ is non-oscillatory, $\lambda_{2}<0$ and $R(r)$ is given by the oscillatory form (10.4.25).

In the event that $\lambda_{2}=k^{2}, \alpha=0$ and one must require that $m=0$ and $F=0$ in (10.4.25) to obtain a bounded but non-null solution. The overall solution is then the plane wave

$$
u(r, \theta, z) \sim e^{ \pm i k z} .
$$

A second special case involving $\alpha=0$ arises when $k^{2}=0$ (so the partial differential equation is Laplace's equation) and $\lambda_{2}=0$ (so there is no $\mathrm{z}$ dependence). The equation for $R$ becomes

$$
\frac{d^{2} R}{d r^{2}}+\frac{1}{r} \frac{d R}{d r}-\frac{m^{2}}{r^{2}} R=0
$$

which has the general solutions

$$
R(r)=\left\{\begin{array}{cc}
G r^{m}+H r^{-m}, & m \neq 0 \\
G+H \ln r, & m=0 .
\end{array}\right.
$$


Forming a superposition of solutions as we did in the examples of Section 10.3 gives us the potential

$$
\psi(r, \theta)=A_{0}+B_{0} \ln r+\sum_{m=1}^{\infty}\left(A_{m} r^{m}+B_{m} r^{-m}\right)\left(C_{m} \cos m \theta+D_{m} \sin m \theta\right)
$$

which we recognize as a full Fourier series in $\theta$. The coefficients $A_{m}, B_{m}, C_{m}$ and $D_{m}, m \geqslant 0$ can be determined by imposing non-homogeneous boundary conditions at two fixed values of $r, \psi(a, \theta)=V_{1}(\theta)$ and $\psi(b, \theta)=V_{2}(\theta)$ for example, and then using the Euler formulae for the Fourier coefficients of the functions $V_{1}(\theta)$ and $V_{2}(\theta)$.

As if (10.4.29) is not complicated enough, a superposition of solutions of the Helmholtz equation with homogeneous boundary conditions at $r=0$ and $r=a$ has the form

$$
\begin{aligned}
& u(r, \theta, z)=\sum_{n=1}^{\infty} \sum_{m=0}^{\infty} J_{m}\left(\alpha_{m n} r\right)\left(A_{m n} \cos m \theta+B_{m n} \sin m \theta\right) \\
& \times\left(C_{m n} \cosh \sqrt{\alpha_{m n}^{2}-k^{2}} z+D_{m n} \sinh \sqrt{\alpha_{m n}^{2}-k^{2}} z\right) .
\end{aligned}
$$

This is a Fourier series in the $\theta$ coordinate as well as a series unlike anything we have seen thus far: an expansion in terms of an infinite set of Bessel functions. Evidently, our knowledge of series representations requires extension if we are to feel comfortable working with cylindrical polars.

What further complications await us when we switch to spherical polars? In spherical coordinates Helmholtz' equation assumes the form

$$
\frac{1}{r} \frac{\partial^{2}}{\partial r^{2}}(r u)+\frac{1}{r^{2} \sin \theta}\left[\frac{\partial}{\partial \theta}\left(\sin \theta \frac{\partial u}{\partial \theta}\right)+\frac{1}{\sin \theta} \frac{\partial^{2} u}{\partial \varphi^{2}}\right]+k^{2} u=0 .
$$

Assuming a separated solution $u=R(r) Y(\theta, \varphi)$, substituting into (10.4.31), and dividing by $u=R Y$, we obtain

$$
\frac{1}{R} \frac{1}{r} \frac{d^{2}}{d r^{2}}(r R)+\frac{1}{r^{2}} \frac{1}{Y \sin \theta}\left[\frac{\partial}{\partial \theta}\left(\sin \theta \frac{\partial Y}{\partial \theta}\right)+\frac{1}{\sin \theta} \frac{\partial^{2} Y}{\partial \varphi^{2}}\right]+k^{2}=0 .
$$

Separating variables yields the equation

$$
\frac{1}{Y} \frac{1}{\sin \theta}\left[\frac{\partial}{\partial \theta}\left(\sin \theta \frac{\partial Y}{\partial \theta}\right)+\frac{1}{\sin \theta} \frac{\partial^{2} Y}{\partial \varphi^{2}}\right]=-\lambda
$$

for the angular dependence of $u(r, \theta, \varphi)$ plus the radial equation

$$
\frac{1}{R} \frac{1}{r} \frac{d^{2}}{d r^{2}}(r R)+k^{2}-\frac{\lambda}{r^{2}}=0
$$

where $\lambda$ is the separation constant. 
We shall start with the angular equation (10.4.33) which we subject to a further separation of variables. Setting $Y=\Theta(\theta) \Phi(\varphi)$, we obtain

$$
\frac{1}{\Theta} \frac{1}{\sin \theta} \frac{d}{d \theta}\left(\sin \theta \frac{d \Theta}{d \theta}\right)+\frac{1}{\sin ^{2} \theta} \frac{1}{\Phi} \frac{d^{2} \Phi}{d \varphi^{2}}+\lambda=0
$$

and hence,

$$
\frac{1}{\Phi} \frac{d^{2} \Phi}{d \varphi^{2}}=-m^{2}, \quad m=0, \pm 1, \pm 2, \ldots
$$

and

$$
\frac{1}{\sin \theta} \frac{d}{d \theta}\left(\sin \theta \frac{d \Theta}{d \theta}\right)+\left(\lambda-\frac{m^{2}}{\sin ^{2} \theta}\right) \Theta=0,
$$

where we have invoked the boundary condition $\Phi(\varphi+2 \pi)=\Phi(\varphi)$ to ensure singlevalued solutions and determine the second separation constant. The corresponding eigensolutions are a linear combination of $\cos m \varphi$ and $\sin m \varphi$ or of $e^{ \pm i m \varphi}$. In this instance we will choose the latter and write

$$
\Phi \equiv \Phi_{m}(\varphi)=A_{m} e^{i m \varphi}+B_{m} e^{-i m \varphi} .
$$

To identify solutions of (10.4.36), we introduce the new variable $x=\cos \theta$ which transforms the equation into a version of Legendre's equation:

$$
\left(1-x^{2}\right) \frac{d^{2} P}{d x^{2}}-2 x \frac{d P}{d x}+\left[\lambda-\frac{m^{2}}{1-x^{2}}\right] P=0 \text { where } P(x)=\Theta\left(\cos ^{-1} x\right) .
$$

Since $\theta$ varies over the range $0 \leqslant \theta \leqslant \pi, x$ has the range $-1 \leqslant x \leqslant 1$. But as we know, the boundary points $x= \pm 1$ are regular singular points of Legendre's equation. Therefore, an obvious boundary condition to impose on the solutions of (10.4.38) is that they be bounded at $x= \pm 1$. This can be satisfied if and only if $\lambda$ is assigned one of the discrete eigenvalues $\lambda=l(l+1), l=0,1,2, \ldots$ with $l \geqslant|m|$; the corresponding eigensolutions are the associated Legendre polynomials, denoted $P_{l}^{m}(x)$. How do these relate to the Legendre polynomials whose acquaintance we made in Chapter 9 ? The answer will be derived in Chapter 11 but here is a preview:

$$
P_{l}^{m}(x)=\left(1-x^{2}\right)^{\frac{m}{2}} \frac{d^{m} P_{l}(x)}{d x^{m}}, \quad m=0,1,2, \ldots l .
$$

The product

$$
Y=\Theta(\theta) \Phi(\varphi)=P_{l}^{m}(\cos \theta) e^{i m \varphi},
$$

with appropriate normalization to be defined later, is called a spherical harmonic. We will have occasion in Chapter 11 to study its properties in some detail. It suffices at present to note that it is an eigenfunction solution of (10.4.33) combined with the periodicity and boundedness conditions and hence, an eigenfunction of the angular part of the partial differential operator $\nabla^{2}$. 
We can now turn our attention to the radial equation (10.4.34) with $\lambda$ set equal to $l(l+1)$ :

$$
\frac{d^{2} R}{d r^{2}}+\frac{2}{r} \frac{d R}{d r}+\left[k^{2}-\frac{l(l+1)}{r^{2}}\right] R=0 .
$$

If $k^{2} \neq 0$, we set $r=\rho / k$ and $R=\frac{1}{\sqrt{\rho}} S$ to transform this equation into

$$
\frac{d^{2} S}{d \rho^{2}}+\frac{1}{\rho} \frac{d S}{d \rho}+\left[1-\frac{(l+1 / 2)^{2}}{\rho^{2}}\right] S=0
$$

which we recognize as Bessel's equation of order $l+1 / 2$. Thus, we conclude that the general solution of the radial equation is

$$
R(r)=A \frac{1}{\sqrt{k r}} J_{l+1 / 2}(k r)+B \frac{1}{\sqrt{k r}} N_{l+1 / 2}(k r)=A^{\prime} j_{l}(k r)+B^{\prime} n_{l}(k r),
$$

where $j_{l}(x) \equiv \sqrt{\frac{\pi}{2 x}} J_{l+1 / 2}(x)$ and $n_{l}(x) \equiv \sqrt{\frac{\pi}{2 x}} N_{l+1 / 2}(x)$ are called spherical Bessel and Neumann functions of order $l$, respectively.

If $k^{2}=0$, which corresponds to Laplace's equation, the radial equation is

$$
\frac{d^{2} R}{d r^{2}}+\frac{2}{r} \frac{d R}{d r}-\frac{l(l+1)}{r^{2}} R=0
$$

which has the general solution

$$
R=A r^{l}+B \frac{1}{r^{l+1}}
$$

Summarizing, the sort of superpositions we can expect in problems with spherical geometry are potentials of the form

$$
\psi(r, \theta, \varphi)=\sum_{l=0}^{\infty} \sum_{m=-l}^{l}\left[A_{l m} r^{l}+B_{l m} r^{-l-1}\right] Y_{l}^{m}(\theta, \varphi)
$$

and waves like

$$
u(r, \theta, \varphi)=\sum_{l=0}^{\infty} \sum_{m=-l}^{l}\left[A_{l m} j_{l}(k r)+B_{l m} n_{l}(k r)\right] Y_{l}^{m}(\theta, \varphi) .
$$

Thus, Fourier series do not figure in the solutions at all when spherical coordinates are used. Rather, we have a double series expansion in terms of the spherical harmonics and so yet another type of series representation to become familiar with. Fortunately, all of these representations are special cases of a Sturm-Liouville eigenfunction expansion and so we can acquire a comprehensive understanding by considering a single eigenvalue problem.

A student of Poisson at the École Polytechnique, Joseph Liouville (1809-1882) contributed widely to mathematics, mathematical physics and astronomy. The Liouville theorem of complex analysis and the Liouville theorem of classical mechanics are both named after him as is the Liouville crater on the moon. He developed Sturm-Liouville theory in collaboration with a colleague at the École Polytecnique, Jacques Sturm (18031855). 


\subsection{The Sturm-Liouville Eigenvalue Problem}

To review, the separation of variables in a partial differential equation results in two or more ordinary differential equations which, when combined with homogeneous boundary conditions, become eigenvalue equations whose solutions correspond to characteristic values of the separation constant(s). Once all of these are known, we use them to express the solution of the original partial differential equation as an eigenfunction expansion and then impose whatever non-homogeneous boundary conditions may be associated with the problem. In the vibrating string problem the eigenfunction expansion is a Fourier sine series which is a type of series that is reasonably familiar to us. But what are the convergence properties of series involving Legendre polynomials or Bessel functions? How do we determine their coefficients? Is there a connection with the theory of Fourier representations?

The most general way of answering these questions is to study the SturmLiouville eigenvalue problem. It consists of solving a differential equation of the form

$$
\mathfrak{L} u(x) \equiv \frac{d}{d x}\left[p(x) \frac{d u(x)}{d x}\right]-q(x) u(x)=-\lambda \rho(x) u(x)
$$

where $\rho(x) \geqslant 0$ on the interval $a \leqslant x \leqslant b$ of the real line and the solution $u(x)$ is subject to (homogeneous) boundary conditions such as $u(a)=u(b)$ and $u^{\prime}(a)=u^{\prime}(b)$, (the periodicity condition is an example of this), or

$$
\begin{array}{r}
\alpha_{1} u+\beta_{1} \frac{d u}{d x}=0 \text { at } x=a \text { and } \\
\alpha_{2} u+\beta_{2} \frac{d u}{d x}=0 \text { at } x=b
\end{array}
$$

where $\alpha_{1}, \beta_{1}, \alpha_{2}$, and $\beta_{2}$ are given constants. The form of the differential operator $\mathfrak{L}$ in (10.5.1) is quite general since after multiplication by a suitable factor any second order linear differential operator can be expressed this way.

The differential equations obtained by separating variables in the preceding Section are all of the Sturm-Liouville type, the separation constants being the eigenvalue parameters $\lambda$. The boundary conditions to go with them, such as boundedness or periodicity, were determined by the requirements of the physics problem in which the equations arise and this is invariably the case.

Changing the boundary conditions can result in a profound change to the eigenvalue spectrum of a differential operator. To illustrate, we shall consider the simple operator $\mathfrak{L} \equiv \frac{d^{2}}{d x^{2}}$. Its Sturm-Liouville equation is

$$
\mathfrak{L} u(x) \equiv \frac{d^{2}}{d x^{2}} u(x)=-\lambda u(x),
$$


corresponding to $p(x) \equiv 1, q(x) \equiv 0, \rho(x) \equiv 1$. We know already that this equation has solutions

$$
u(x)=\left\{\begin{array}{cl}
A \cos \sqrt{\lambda} x+B \sin \sqrt{\lambda} x & \text { if } \lambda>0 \\
A \cosh \sqrt{-\lambda} x+B \sinh \sqrt{-\lambda} x & \text { if } \lambda<0 \\
A x+B & \text { if } \lambda=0
\end{array}\right.
$$

So, if the boundary conditions are

- $u(x+2 \pi)=u(x)$ then,

$$
\lambda=m^{2} \text { and } u_{m}(x)=A_{m} \cos m x+B_{m} \sin m x, \quad m=0,1,2, \ldots,
$$

- $u(0)=0$ and $u(b)=0$ then,

$$
\lambda=\frac{n^{2} \pi^{2}}{b^{2}} \text { and } u_{n}(x)=A_{n} \sin \frac{n \pi x}{b}, \quad n=1,2,3, \ldots,
$$

- $u^{\prime}(0)=0$ and $|u(\infty)|<\infty$ then,

$$
\lambda=k^{2} \text { and } u_{k}(x)=A_{k} \cos k x, \quad 0 \leqslant k<\infty,
$$

- $|u( \pm \infty)|<\infty$ then,

$$
\lambda=k^{2} \text { and } u_{k}(x)=A_{k} e^{i k x}, \quad-\infty<k<\infty .
$$

The multiplicative constants in these expressions are determined by some nonlinear normalization condition such as

$$
\int_{0}^{b}\left|u_{n}(x)\right|^{2} d x=1
$$

\begin{tabular}{|c|c|c|c|c|c|c|}
\hline Equation & $\begin{array}{l}\text { Boundary } \\
\text { Conditions }\end{array}$ & Eigenfunctions & Eigenvalues & $\rho(x)$ & $p(x)$ & $q(x)$ \\
\hline Legendre & $|u( \pm 1)|^{2}<\infty$ & $\underset{\substack{l=0,1,2, \ldots \\
P_{l}(x)}}{ }$ & $l(l+1)$ & 1 & $1-x^{2}$ & 0 \\
\hline $\begin{array}{l}\text { Associated } \\
\text { Legendre }\end{array}$ & ditto & $P_{l}^{m}(x)$ & $l(l+1)$ & 1 & $1-x^{2}$ & $\frac{m^{2}}{1-x^{2}}$ \\
\hline Bessel & $|\boldsymbol{u}(0)|<\infty$ & $J_{m}(x)$ & 1 & $x$ & $x$ & $\frac{m^{2}}{x}$ \\
\hline $\begin{array}{l}\text { Spherical } \\
\text { Bessel }\end{array}$ & ditto & $j_{l}(x)$ & 1 & $x^{2}$ & $x^{2}$ & $l(l+1)$ \\
\hline
\end{tabular}

Some other Sturm-Liouville problems encountered in Section 10.4 are reviewed in the following table (Table 10.1).

Table 10.1: Sturm-Liouville Problems in Section 10.4 
The Sturm-Liouville eigenvalue problem is an infinite-dimensional analogue of the matrix eigenvalue problem

$$
M u=\lambda u,
$$

where $M$ is an $n \times n$ matrix and $u$ is an $n$-dimensional column vector, encountered in connection with finite dimensional vector spaces. In both cases there are solutions $u_{n}$ only for certain values of the eigenvalue $\lambda_{n}$. These are called the eigenvectors of $\mathrm{M}$ while in the case of $\mathfrak{L}$,they are the eigenfunctions corresponding to the particular choice of boundary conditions that accompany the equation. Significantly, like the eigenvectors of matrices, the eigenfunctions of $\mathfrak{L}$ can be used as basis vectors spanning a type of vector space in which the vectors are functions. Such function spaces are generally infinite dimensional corresponding either to a countable infinity or to a continuum of eigenfunctions and eigenvalues. An example of the former is the space consisting of all square integrable functions defined on a finite interval $a \leqslant x \leqslant b$. A continuum normally arises when one or both of the end-points is at infinity.

In a conventional vector space each vector is an ordered n-tuple of numbers,

$$
\boldsymbol{a} \equiv \mid a>\equiv\left(a_{1}, a_{2}, \ldots, a_{n}\right) .
$$

The numbers can be real or imaginary. The number of dimensions, $n$, can be finite or infinite. Various operations such as addition, subtraction and multiplication by a scalar are defined as is the operation of scalar product,

$$
\boldsymbol{a} \cdot \boldsymbol{b} \equiv<a \mid b>\equiv \sum_{i=1} a_{i}^{\star} b_{i}
$$

The ordering is discrete and even if the number of dimensions is infinite, it is a "countable infinity".

Functions also provide ordered sets of numbers although now the ordering is continuous: $f(x), a \leqslant x \leqslant b$, denotes an ordered continuum of numbers. Thus, the set of all functions which satisfy certain behavioural conditions on an interval of the real line, $a \leqslant x \leqslant b$, can define a vector space called a function space. An example is the set of functions which are square integrable. The scalar product is defined in analogy with the definition for a conventional vector space,

$$
\langle u \mid v\rangle \equiv \sum_{\text {all components }} u^{\star}(x) v(x) \equiv \int_{a}^{b} u^{\star}(x) v(x) \rho(x) d x .
$$

Here, $\rho(x)$ is a weight function that determines how one counts "components" as $x$ varies along the real line from $a$ to $b: \rho(x) d x=$ the number of "components" in the interval $d x$ about $x$.

In conventional vector spaces it is convenient to define a basis (or bases) of orthogonal unit vectors $\boldsymbol{e}_{i}, i=1,2, \ldots n$ with $\boldsymbol{e}_{i} \cdot \boldsymbol{e}_{j}=\delta_{i, j}$ so that each vector $\boldsymbol{a}$ can be 
expressed as

$$
\boldsymbol{a}=\sum_{i=1} a_{i} \boldsymbol{e}_{i}, a_{j}=\boldsymbol{e}_{j} \cdot \boldsymbol{a}(j=1,2, \ldots) .
$$

Notice that $\boldsymbol{e}_{i} \cdot \boldsymbol{e}_{j}=\delta_{i, j}$ captures both orthogonality and normalization. The corresponding expressions in "ket" notation are

$$
\left|a>=\sum_{i=1} a_{i}\right| e_{i}>, \quad a_{j}=<e_{j} \mid a>\text { and }\left\langle e_{i}\right| e_{j}>=\delta_{i, j} .
$$

When all vectors in a space can be so expressed the basis is said to be complete with respect to the space. (The adjective complete should have a familiar ring to it.)

The same is true of function spaces. One can determine basis vectors (functions) $u_{n}(x)$ which are orthonormal,

$$
<u_{m} \mid u_{n}>=\int_{a}^{b} u_{m}^{\star}(x) u_{n}(x) \rho(x) d x=\delta_{m, n},
$$

and which are complete with respect to the space. That means that each $f(x)$ in the space can be expanded in the series

$$
f(x)=\sum_{m=1}^{\infty} c_{m} u_{m}(x) \text { where } c_{m}=\int_{a}^{b} u_{m}^{\star}(x) f(x) \rho(x) d x .
$$

Now we remember where we have encountered the term complete before. It was in connection with Parseval's equation and the representation of functions that are square integrable on $-\pi \leqslant x \leqslant \pi$ in terms of the Fourier functions $\{\cos n x, \sin n x\}$.

Having digressed into the algebraic perspective on series representations, let us return to the analysis of the Sturm-Liouville problem. Its solutions have some general properties of key importance. These follow in large part from general properties possessed by the Sturm-Liouville operator $\mathfrak{L}$. Specifically, suppose that $u(x)$ and $v(x)$ are arbitrary twice differentiable functions. For increased generality, we shall take them to be complex. We write

$$
\begin{gathered}
v^{\star}(x) \mathfrak{L} u(x) \equiv v^{\star}(x) \frac{d}{d x}\left[p(x) \frac{d u(x)}{d x}\right]-v^{\star}(x) q(x) u(x), \\
u(x)(\mathfrak{L} v(x))^{\star} \equiv u(x) \frac{d}{d x}\left[p(x) \frac{d v^{\star}(x)}{d x}\right]-u(x) q(x) v^{\star}(x),
\end{gathered}
$$

take the difference, and then integrate by parts to obtain

$$
\int_{a}^{b} v^{\star}(\mathfrak{L} u) d x-\int_{a}^{b} u(\mathfrak{L} v)^{\star} d x=\left.p(x)\left[v^{\star}(x) \frac{d u(x)}{d x}-u(x) \frac{d v^{\star}(x)}{d x}\right]\right|_{x=a} ^{x=b} .
$$

This is an instance of the generalized Green's identity that we encountered in Section 9.9 and its appearance here tells us that the Sturm-Liouville operator $\mathfrak{L}$ is selfadjoint wth respect to the weight function $w(x)=1$. 
Note that if the functions $u(x)$ and $v(x)$ both satisfy the homogeneous boundary conditions

$$
\begin{aligned}
& \alpha_{1} y(a)+\beta_{1} y^{\prime}(a)=0 \\
& \alpha_{2} y(b)+\beta_{2} y^{\prime}(b)=0
\end{aligned}
$$

or the conditions

$$
y(a)=y(b) \text { and } y^{\prime}(a)=y^{\prime}(b) \text { together with } p(a)=p(b),
$$

the surface term on the right hand side of (10.5.18) vanishes and we obtain the Green's identity for self-adjoint operators

$$
\int_{a}^{b} v^{\star}(x)(\mathfrak{L} u(x)) d x=\int_{a}^{b} u(x)(\mathfrak{L} v(x))^{\star} d x .
$$

In algebraic terms, imposing homogeneous boundary conditions on a set of functions $u(x)$ defines a function space; a self-adjoint differential operator $\mathfrak{L}$ then defines a Hermitian operator on that space.

The fact that the Sturm-Liouville operator is self-adjoint has important consequences for its eigenfunctions and eigenvalues. Suppose that we have two different eigenfunctions $u_{n}(x)$ and $u_{m}(x)$ corresponding to the eigenvalues $\lambda_{n}$ and $\lambda_{m}, \lambda_{n} \neq \lambda_{m}$ :

$$
\begin{gathered}
\mathfrak{L} u_{n}(x)=-\lambda_{n} \rho(x) u_{n}(x), \\
\mathfrak{L} u_{m}(x)=-\lambda_{m} \rho(x) u_{m}(x) .
\end{gathered}
$$

We shall allow for the possibility of complex eigenfunctions and even complex eigenvalues but, by definition, $\rho(x)$ and $\mathfrak{L}$ are real. Multiplying (10.5.23) by $u_{m}^{\star}(x)$ and the complex conjugate of (10.5.24) by $u_{n}(x)$, subtracting and integrating, we find

$$
\int_{a}^{b}\left[u_{m}^{\star}(x) \mathfrak{L} u_{n}(x)-u_{n}(x) \mathfrak{L} u_{m}^{\star}(x)\right] d x=-\left(\lambda_{n}-\lambda_{m}^{\star}\right) \int_{a}^{b} u_{m}^{\star}(x) u_{n}(x) \rho(x) d x .
$$

Since $u_{n}(x)$ and $u_{m}(x)$ are eigenfunctions, they satisfy homogeneous boundary conditions and, as we have seen, that means that the left hand side of (10.5.25) must vanish. Thus,

$$
\left(\lambda_{n}-\lambda_{m}^{\star}\right) \int_{a}^{b} u_{m}^{\star}(x) u_{n}(x) \rho(x) d x=0
$$

If $n=m$, the integral cannot vanish because both $\rho(x)$ and $\left|u_{m}(x)\right|^{2}$ are nonnegative. Therefore, we conclude that $\lambda_{m}^{\star}=\lambda_{m}$; all the eigenvalues of the SturmLiouville operator are real. 
If $n \neq m$ and $\lambda_{n} \neq \lambda_{m}$, we conclude that

$$
\int_{a}^{b} u_{m}^{\star}(x) u_{n}(x) \rho(x) d x=0 .
$$

Two functions $u_{n}(x)$ and $u_{m}(x)$ satisfying a condition like (10.5.27) are said to be orthogonal with respect to the weight function $\rho(x)$. In other words, the functions $\left\{u_{m}(x)\right\}$ comprise an orthogonal set of vectors in a function space where the scalar product between two vectors $u(x)$ and $v(x)$ is defined to be

$$
\boldsymbol{v}^{\star} \cdot \boldsymbol{u} \equiv\langle v| u>\equiv \int_{a}^{b} v^{\star}(x) u(x) \rho(x) d x
$$

If we normalize the eigenfunctions by requiring

$$
\int_{a}^{b}\left|u_{m}(x)\right|^{2} \rho(x) d x \equiv\left\|u_{m}\right\|^{2}=1,
$$

we obtain an orthonormal set and (10.5.27) and (10.5.29) combine to read

$$
\int_{a}^{b} u_{m}^{\star}(x) u_{n}(x) \rho(x) d x=\delta_{m, n} .
$$

It is also possible to have $n \neq m$, but $\lambda_{n}=\lambda_{m}=\lambda$. If this happens, we say that $\lambda$ is degenerate and equation (10.5.26) no longer requires the corresponding eigenfunctions to be orthogonal.. However, we can always choose or construct them to be orthogonal by forming orthogonal linear combinations.

The most important consequence of the self-adjoint character of the SturmLiouville operator is one that we shall state without proof. (The proof can be found in a variety of analysis texts such as Courant and Hilbert or E.C. Titchmarsh.) Its statement is as follows: the eigenfunctions of a Sturm-Liouville operator comprise a complete set of functions. Algebraically, this means that they span the function space on which they are defined and can be used as basis vectors for that space. Thus, any other function (vector) $f(x)$ in the space can be expanded in terms of them,

$$
f(x)=\sum_{m} c_{m} u_{m}(x)
$$

where the coefficients $c_{m}$ are the "components of $f(x)$ along the 'unit' vectors $u_{m}(x)$ ",

$$
c_{m}=<u_{m} \mid f>=\int_{a}^{b} u_{m}^{\star}\left(x^{\prime}\right) f\left(x^{\prime}\right) \rho\left(x^{\prime}\right) d x^{\prime} .
$$


Keep in mind that we have normalized the functions $\left\{u_{m}(x)\right\}$. If that were not the case, (10.5.32) would become

$$
c_{m}=\frac{\int_{a}^{b} u_{m}^{\star}\left(x^{\prime}\right) f\left(x^{\prime}\right) \rho\left(x^{\prime}\right) d x^{\prime}}{\int_{a}^{b}\left|u_{m}\left(x^{\prime}\right)\right|^{2} \rho\left(x^{\prime}\right) d x^{\prime}} .
$$

In general, the eigenfunction expansion in (10.5.31) is an infinite series and so the statement of completeness implies a statement about the convergence of the series. Since the Fourier functions $\left\{\frac{1}{\sqrt{T}} \exp \left[i \frac{2 \pi m}{T} x\right]\right\}$ are Sturm-Liouville eigenfunctions, it is not surprising that the convergence properties of Fourier series characterize those of all such eigenfunction expansions. In fact, (10.5.31) is sometimes referred to as a generalized Fourier series and the $c_{m}$ as generalized Fourier coefficients. In particular, if $f(x)$ is square integrable with respect to $\rho(x)$ over $a \leqslant x \leqslant b$, then we are assured that the series

$$
\sum_{m=1}^{\infty} c_{m} u_{m}(x) \text { with } c_{m}=\int_{a}^{b} u_{m}^{\star}\left(x^{\prime}\right) f\left(x^{\prime}\right) \rho\left(x^{\prime}\right) d x^{\prime}
$$

must at least converge in the mean to $f(x)$ and therefore,

$$
<\left.f\left|f>=\int_{a}^{b}\right| f(x)\right|^{2} \rho(x) d x=\sum_{m=1}^{\infty}\left|c_{m}\right|^{2}=\sum_{m=1}^{\infty}<f\left|u_{m}><u_{m}\right| f>.
$$

Equation (10.5.35) is called a completeness relation. Having it hold for all vectors $f(x)$ in a function space defined over $a \leqslant x \leqslant b$ is a necessary and sufficient condition for the set $\left\{u_{m}(x)\right\}$ to be complete with respect to that space.

We encountered convergence in the mean in connection with Fourier series. To remind, it means that if $S_{N}$ is the $N$ th partial sum of the series,

$$
S_{N}=\sum_{m=1}^{N} c_{m} u_{m}(x)
$$

then,

$$
\lim _{N \rightarrow \infty} \int_{a}^{b}\left|f(x)-S_{N}(x)\right|^{2} \rho(x) d x=0 .
$$

This does not imply point-wise convergence let alone uniform convergence of the series. However, one can prove that if we further restrict $f(x)$ so that it is piecewise continuous with a square integrable first derivative over $a \leqslant x \leqslant b$, the eigenfunction expansion (10.5.34) converges absolutely and uniformly to $f(x)$ in all sub-intervals free of 
points of discontinuity and at the points of discontinuity it converges to the arithmetic mean of the right and left hand limits of $f(x)$. If there are no points of discontinuity and in addition, if $f(x)$ satisfies the boundary conditions imposed on $\left\{u_{m}(x)\right\}$, the expansion converges uniformly throughout $a \leqslant x \leqslant b$.

In physics texts the completeness relation is often complemented by and confused with an equation called the closure relation. Substituting (10.5.32) into (10.5.31) and reversing the order of summation and integration, we have

$$
f(x)=\int_{a}^{b}\left[\rho\left(x^{\prime}\right) \sum_{m} u_{m}(x) u_{m}^{\star}\left(x^{\prime}\right)\right] f\left(x^{\prime}\right) d x^{\prime}
$$

for an arbitrary function $f(x)$. Comparing this with the defining property of Dirac delta functions,

$$
\int_{a}^{b} \delta\left(x^{\prime}-x\right) f\left(x^{\prime}\right) d x^{\prime}=f(x), a \leqslant x \leqslant b,
$$

we conclude that

$$
\rho\left(x^{\prime}\right) \sum_{m} u_{m}(x) u_{m}^{\star}\left(x^{\prime}\right)=\delta\left(x^{\prime}-x\right)
$$

\subsection{A Convenient Notation (And Another Algebraic Digression)}

We have defined the scalar product in a complex function space as

$$
\boldsymbol{v}^{\star} \cdot \boldsymbol{u}=\langle v| u>=\int_{a}^{b} v^{\star}(x) u(x) \rho(x) d x,
$$

where $\rho(x)$ is a suitable weight function. This is a generalization of the expression

$$
<a \mid b>=\boldsymbol{a}^{\star} \cdot \boldsymbol{b}=\sum_{j=1}^{N} a_{j}^{\star} b_{j}
$$

for the scalar product in an N-dimensional complex number vector space in which an orthonormal basis has been chosen. In a function space, the vector $\mid u>$ corresponds to the entire set (or continuum) of values assumed by a function $u(x)$ for $a \leqslant x \leqslant b$. Therefore, it is convenient to consider the number $u(x)$ for a specific value of $x$ to be the $x^{\text {th }}$ component of the vector $\mid u>$. This implies the existence of a set of basis vectors $\mid x>, a \leqslant x \leqslant b$, such that

$$
u(x) \equiv<x \mid u>
$$


The continuity of $x$ gives rise to difficulties in defining the normalization of $\mid x>$. We assume, of course, that two distinct basis vectors $\mid x>$ and $\mid x^{\prime}>$ are orthogonal,

$$
<x^{\prime} \mid x>=0 \text { for } x^{\prime} \neq x .
$$

Moreover, we assume that the analogue of the familiar decomposition of a vector in terms of an orthonormal basis,

$$
\left|a>=\sum_{j}^{N} a_{j}\right| e_{j}>, \quad a_{k}=<e_{k} \mid a>(k=1,2, \ldots, N),
$$

is

$$
\left|u>=\int_{a}^{b} d x \rho(x) u(x)\right| x>
$$

This means the scalar product of $\mid u>$ with $\mid x^{\prime}>$ can be written

$$
<x^{\prime}\left|u>=u\left(x^{\prime}\right)=\int_{a}^{b} d x \rho(x) u(x)<x^{\prime}\right| x>
$$

which implies that $\rho(x)<\chi^{\prime} \mid x>$ has the properties of a Dirac $\delta$-function. Evidently, we cannot normalize $\mid x>$ to unity. Rather, the analogue of $\left\langle e_{j}\right| e_{k}>=\delta_{j, k}=\left\{\begin{array}{l}1 \text { if } j=k \\ 0 \text { if } j \neq k\end{array}\right.$ is

$$
<x \mid x^{\prime}>=\frac{1}{\sqrt{\rho(x) \rho\left(x^{\prime}\right)}} \delta\left(x-x^{\prime}\right)=\frac{1}{\rho(x)} \delta\left(x-x^{\prime}\right)=\frac{1}{\rho\left(x^{\prime}\right)} \delta\left(x-x^{\prime}\right),
$$

which is not so surprising once we remember that the Dirac $\delta$-function is a continuous variable analogue of the Kronecker $\delta$-function $\delta_{j, k}$.

As we saw in the preceding Section, our function space can also have an enumerable orthonormal basis consisting of vectors $\mid u_{m}>$ represented by the functions $u_{m}(x)$

$$
u_{m}(x)=<x \mid u_{m}>, \quad m=1,2, \ldots
$$

The closure relation satisfied by these functions is (see (10.5.37))

$$
\frac{1}{\rho\left(x^{\prime}\right)} \delta\left(x^{\prime}-x\right)=\sum_{m=1}^{\infty} u_{m}(x) u_{m}^{\star}\left(x^{\prime}\right)
$$

Using the normalization equation (10.6.5), we can rewrite this as

$$
<x\left|x^{\prime}>=\sum_{m=1}^{\infty}<x\right| u_{m}><u_{m}\left|x^{\prime}>=<x\right|\left(\sum_{m=1}^{\infty}\left|u_{m}><u_{m}\right|\right) \mid x^{\prime}>.
$$


Since $\mid x>$ and $\mid x^{\prime}>$ are arbitrary basis vectors, the object in brackets must be the identity operator $\mathbb{I}$. Thus, an alternative expression of closure is

$$
\mathbb{I}=\sum_{m=1}^{\infty}\left|u_{m}><u_{m}\right|
$$

The analogous relation for the basis $\mid x>, a \leqslant x \leqslant b$, is

$$
\mathbb{I}=\int_{a}^{b} d x \rho(x)|x><x|
$$

\subsection{Fourier Series and Transforms as Eigenfunction Expansions}

The most familiar examples of complete sets are the Fourier functions

$$
\frac{1}{\sqrt{T}} \exp \left[i \frac{2 \pi m}{T} x\right], \quad m=0, \pm 1, \pm 2, \ldots, a \leqslant x \leqslant a+T
$$

and

$$
\frac{1}{\sqrt{2 \pi}} e^{-i k x}, \quad-\infty<k<\infty,-\infty<x<\infty
$$

As we have seen already, the first of these is comprised of the eigenfunction solutions of the Sturm-Liouville equation

$$
\frac{d^{2}}{d x^{2}} u(x)=-\lambda u(x), \quad a \leqslant x \leqslant a+T
$$

subject to the periodic boundary condition $u(x)=u(x+T)$. The corresponding eigenvalues are $\lambda=\left(\frac{2 \pi m}{T}\right)^{2}$. This discrete set is called the spectrum of $\mathfrak{L} \equiv \frac{d^{2}}{d x^{2}}$ when applied to functions which satisfy this boundary condition. The orthogonality relation satisfied by these eigenfunctions is

$$
\int_{a}^{a+T} u_{m}^{\star}(x) u_{n}(x) d x=\delta_{m, n}
$$

which also tells us that they are normalized to unity.

An eigenfunction expansion of a function $f(x)$ in terms of this basis provides a Fourier series representation:

$$
f(x)=\sum_{m=-\infty}^{\infty} c_{m} \frac{1}{\sqrt{T}} \exp \left[i \frac{2 \pi m}{T} x\right]=\frac{a_{0}}{2}+\sum_{m=0}^{\infty}\left[a_{m} \cos \left(\frac{2 \pi m}{T}\right)+b_{m} \sin \left(\frac{2 \pi m}{T}\right)\right],
$$


with

$$
c_{m}=\left\langle u_{m}\right| f>=\frac{1}{\sqrt{T}} \int_{a}^{a+T} \exp \left[-i \frac{2 \pi m}{T} x\right] f(x) d x .
$$

The completeness relation for the Fourier functions is

$$
\int_{a}^{a+T}|f(x)|^{2} d x=\sum_{m=-\infty}^{\infty}\left|c_{m}\right|^{2}
$$

or,

$$
\frac{a_{0}^{2}}{2}+\sum_{m=1}^{\infty}\left[a_{m}^{2}+b_{m}^{2}\right]=\frac{2}{T} \int_{a}^{a+T}|f(x)|^{2} d x .
$$

The latter expression is known as Parseval's equation in the theory of Fourier series.

At this point the reader may wish to return to our analysis of the solution of the stretched string problem since it centres upon the identification of a Fourier sine series as an eigenfunction expansion.

Suppose that the range of $x$ is the entire real line so that (10.7.3) is replaced by

$$
\frac{d^{2}}{d x^{2}} u(x)=-\lambda u(x), \quad-\infty<x<\infty
$$

and the periodic boundary condition is replaced by

$$
|u( \pm \infty)|<\infty .
$$

As we have seen, the eigenfunctions are now

$$
u_{k}(x)=e^{-i k x}, \quad-\infty<k<\infty
$$

and the corresponding eigenvalues are $\lambda=k^{2}$. Notice that $\mathfrak{L} \equiv \frac{d^{2}}{d x^{2}}$ now has a continuous spectrum. The eigenfunctions' orthogonality relation is

$$
\int_{-\infty}^{\infty} e^{i k x} e^{-i k^{\prime} x} d x=2 \pi \delta\left(k-k^{\prime}\right)
$$

Thus, normalizing the eigenfunctions, we arrive at the form given in (10.7.2):

$$
u_{k}(x)=\frac{1}{\sqrt{2 \pi}} e^{-i k x}, \quad-\infty<k<\infty .
$$

An eigenfunction expansion of a function $f(x)$ defined on $-\infty<x<\infty$ in terms of this basis is given by the continuous sum

$$
f(x)=\int_{-\infty}^{\infty} F(k) u_{k}(x) d k
$$


where

$$
F(k)=<u_{k} \mid f>=\int_{-\infty}^{\infty} u_{k}^{\star}\left(x^{\prime}\right) f\left(x^{\prime}\right) d x^{\prime}
$$

Substituting for $u_{k}(x)$, we see that $F(k)$ is the Fourier transform of $f(x)$,

$$
F(k)=\frac{1}{\sqrt{2 \pi}} \int_{-\infty}^{\infty} e^{i k x^{\prime}} f\left(x^{\prime}\right) d x^{\prime} \equiv \mathcal{F}\{f(x)\},
$$

and (10.7.12) is a Fourier integral representation of $f(x)$ :

$$
f(x)=\frac{1}{2 \pi} \int_{-\infty}^{\infty} e^{-i k x} F(k) d k \equiv \mathcal{F}^{-1}\{F(k)\} .
$$

The completeness relation in this case reads

$$
\int_{-\infty}^{\infty}|f(x)|^{2} d x=\int_{-\infty}^{\infty}|F(k)|^{2} d k
$$

which is a result known as Plancherel's Theorem in the theory of Fourier transforms. The closure relation is

$$
\int_{-\infty}^{\infty} u_{k}(x) u_{k}^{*}\left(x^{\prime}\right) d k=\frac{1}{2 \pi} \int_{-\infty}^{\infty} e^{-i k\left(x-x^{\prime}\right)} d k=\delta\left(x-x^{\prime}\right) .
$$

We shall now consider a concrete problem involving a continuous eigenvalue spectrum. Four large conducting plates are arranged with the electrostatic potentials shown in the diagram below.

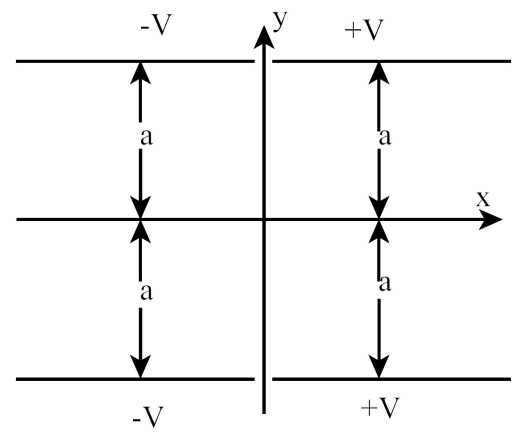

The size of the plates is much larger than the separation $2 a$ and so they can be treated as though they extend to infinity in the $\mathrm{x}$ - and $\mathrm{z}$-directions. We wish to find the 
electrostatic potential in the region between the plates. That means we wish to solve Laplace's equation

$$
\frac{\partial^{2} \psi}{\partial x^{2}}+\frac{\partial^{2} \psi}{\partial y^{2}}+\frac{\partial^{2} \psi}{\partial z^{2}}=0
$$

subject to boundary conditions at the $x$ - and $y$-boundaries but not at the $z$-boundaries. In fact, the symmetry of the problem tells us that there is no z-dependence at all.

Evidently, the conditions at the y-boundaries are

$$
\psi(x, \pm a)=V \text { for } x>0 \text { and } \psi(x, \pm a)=-V \text { for } x<0
$$

which implies, among other things, that $\psi(x, y)$ is an even function of $y$.

Formulating the conditions at the $x$-boundaries requires a little more thought. We note that the conditions in (10.7.19) are odd with respect to $x \rightarrow-\chi$ :

$$
\psi(-x, \pm a)=-\psi(x, \pm a) .
$$

This must also be true for all other values of $y$, that is $\psi(-x, y)=-\psi(x, y)$ for $-a \leqslant y \leqslant$ a. Therefore, we must have the following condition at $x=0$ :

$$
\psi(0, y)=0 \text { for all } y \text { in }-a \leqslant y \leqslant a .
$$

The other x-boundaries are at infinity where we can require

$$
\lim _{x \rightarrow \pm \infty}|\psi(x, y)|<\infty .
$$

Since the conditions at the $\mathrm{x}$-boundaries are homogeneous, we will begin our solution of Laplace's equation by eliminating the derivative with respect to $\mathrm{x}$. This means expanding $\psi(x, y)$ in terms of the eigenfunctions of $\mathfrak{L}=\frac{d^{2}}{d x^{2}}$ that satisfy the boundary conditions $X(0)=0$ and $|X( \pm \infty)|<\infty$. The boundedness requirement means that we have to rule out the possibility that $\lambda<0$ since that corresponds to the exponential solutions $\exp ( \pm \sqrt{-\lambda} x)$ of the equation

$$
\frac{d^{2} X}{d x^{2}}=-\lambda X
$$

The condition at $x=0$ further eliminates the possibility of $\lambda=0$ and of the cosine solution when $\lambda>0$. This leaves us with the eigensolutions $X_{k}(x)=\sin k x, 0 \leqslant k<\infty$ and eigenvalues $\lambda=k^{2}$.

Since the only restriction placed on $k$ is that it be real and positive-definite, we have obtained a continuous eigenvalue spectrum and the eigenfunctions $X_{k}(x)$ comprise a non- denumerably infinite set. Of course we know that when normalized to become $\left\{\sqrt{\frac{2}{\pi}} \sin k x\right\}$ this set of eigenfunctions provides the complete, orthonormal basis for Fourier sine transform representations. Therefore, we can represent $\psi(x, y)$ by the uniformly convergent eigenfunction expansion

$$
\psi(x, y)=\sqrt{\frac{2}{\pi}} \int_{0}^{\infty} \Psi(k, y) \sin k x d k
$$


where the expansion coefficients $\Psi(k, y)$ comprise the Fourier sine transform of $\psi(x, y)$ :

$$
\Psi(k, y)=\mathcal{F}_{S}\{\psi(x, y)\} .
$$

Substituting the representation (10.7.22) into Laplace's equation and interchanging the order of differentiation and integration gives us

$$
\sqrt{\frac{2}{\pi}} \int_{0}^{\infty}\left[-k^{2} \Psi(k, y)+\frac{\partial^{2} \Psi}{\partial y^{2}}\right] \sin k x d k=0 .
$$

In other words, the PDE has been reduced to its Fourier sine transform

$$
\frac{\partial^{2} \Psi}{\partial y^{2}}-k^{2} \Psi(k, y)=0 .
$$

Solving this equation and using the fact that $\Psi(k, y)$ is an even function of $y$ (because $\psi(x, y)$ is even) we obtain

$$
\Psi(k, y)=C(k) \cosh k y .
$$

To find the remaining unknown $C(k)$ we impose the boundary condition $\psi(x, a)=V$; that is, we require that

$$
\Psi(k, a)=\mathcal{F}_{S}\{V\}=\sqrt{\frac{2}{\pi}} \frac{V}{k} .
$$

Thus,

and our final solution is

$$
C(k)=\sqrt{\frac{2}{\pi}} \frac{V}{k} \frac{1}{\cosh k a}
$$

$$
\psi(x, y)=\frac{2 V}{\pi} \int_{0}^{\infty} \frac{\cosh k y}{\cosh k a} \frac{\sin k x}{k} d k .
$$

This demonstrates that using integral transforms is completely equivalent to performing an eigenfunction expansion when the eigenfunctions correspond to a continuous eigenvalue spectrum.

\subsection{Normal Mode (or Initial Value) Problems}

Having started our discussion of boundary value problems with a vibrating string, we shall conclude with a vibrating membrane (or drum head). But first, we shall pursue some theoretical considerations that are relevant to any system that is set in motion via an action that is expressible by means of non-homogeneous initial conditions.

The equation of motion of such a system will typically be one of

$$
\nabla^{2} \psi(\boldsymbol{r}, t)=\frac{1}{D} \frac{\partial \psi}{\partial t} \text { or } \nabla^{2} \psi(\boldsymbol{r}, t)=\frac{1}{c^{2}} \frac{\partial^{2} \psi}{\partial t^{2}}
$$


and the problem will be to solve it within a region $V$ bounded by a surface $S$ subject to time independent boundary conditions on $S$ and to initial conditions that specify $\psi$ and in the case of the wave equation $\frac{\partial \psi}{\partial t}$ throughout $V$ at $t=0$.

The most efficacious way of proceeding is to set

$$
\psi(\boldsymbol{r}, t)=\psi_{1}(\boldsymbol{r})+\psi_{2}(\boldsymbol{r}, t)
$$

where

1. $\nabla^{2} \psi_{1}(\boldsymbol{r})=0$ and $\psi_{1}(\boldsymbol{r})$ satisfies the same boundary conditions on $S$ as does $\psi(\boldsymbol{r}, t)$

2. $\nabla^{2} \psi_{2}(\boldsymbol{r}, t)=\frac{1}{D} \frac{\partial \psi_{2}}{\partial t}$ or $\nabla^{2} \psi_{2}(\boldsymbol{r}, t)=\frac{1}{c^{2}} \frac{\partial^{2} \psi_{2}}{\partial t^{2}}$ and $\psi_{2}(\boldsymbol{r}, t)$ satisfies homogeneous boundary conditions on $S$ and the same initial conditions as does $\psi(r, t)$.

We have seen how one goes about solving for $\psi_{1}(\boldsymbol{r})$ in either Cartesian, cylindrical or spherical coordinates. Therefore, we can focus on the initial value problem associated with $\psi_{2}(\boldsymbol{r}, t)$.

We already know from Section 10.3 how to separate the time dependence. It results in separated solutions of the form

$$
\psi_{2}(\boldsymbol{r}, t)=e^{-D k^{2} t} u(\boldsymbol{r}) \text { or } \psi_{2}(\boldsymbol{r}, t)=\left\{\begin{array}{l}
\cos k c t \\
\sin k c t
\end{array}\right\} u(\boldsymbol{r})
$$

depending on which PDE we are solving. Moreover, in both cases, the time-independent function $u(\boldsymbol{r})$ is required to be a solution of

$$
\nabla^{2} u(\boldsymbol{r})+k^{2} u(\boldsymbol{r})=0
$$

which when accompanied by homogeneous boundary conditions on $S$ is a multidimensional Sturm-Liouville eigenvalue problem. Denoting its eigenfunctions and eigenvalues by $u_{n}(\boldsymbol{r})$ and $k_{n}^{2}$ respectively, we can assert that the former comprise a complete, orthogonal set of functions. In other words,

$$
\int_{V} u_{n}^{\star}(\boldsymbol{r}) u_{m}(\boldsymbol{r}) d V=0 \text { if } n \neq m
$$

and, any function $f(\boldsymbol{r})$ that is square integrable over $V$ can be represented by the (convergent) series

$$
f(\boldsymbol{r})=\sum_{n} c_{n} u(\boldsymbol{r})
$$

where

$$
c_{n}=\frac{\int_{V} u_{n}^{\star}(\boldsymbol{r}) f(\boldsymbol{r}) d V}{\int_{V}\left|u_{n}(\boldsymbol{r})\right|^{2} d V} .
$$


This means that there is an infinite set of solutions of the diffusion and wave equations which satisfy homogeneous boundary conditions on $S$. Each solution has a characteristic time dependence and collectively, they are called the normal modes of the system in question. Forming superpositions of them, we can express any other solution of the diffusion or wave equation as

$$
\psi_{2}(\boldsymbol{r}, t)=\sum_{n} c_{n} e^{-D k_{n}^{2} t} u_{n}(\boldsymbol{r}) \text { or } \sum_{n}\left[a_{n} \cos k_{n} c t+b_{n} \sin k_{n} c t\right] u_{n}(\boldsymbol{r})
$$

respectively. A complete determination of the solution is then made by imposing the initial conditions via an application of (10.8.7):

$$
\begin{gathered}
c_{n}=\frac{\int_{V} u_{n}^{\star}(\boldsymbol{r}) \psi_{2}(\boldsymbol{r}, 0) d V}{\int_{V}\left|u_{n}(\boldsymbol{r})\right|^{2} d V}, \text { or } \\
a_{n}=\frac{\int_{V} u_{n}^{\star}(\boldsymbol{r}) \psi_{2}(\boldsymbol{r}, 0) d V}{\int_{V}\left|u_{n}(\boldsymbol{r})\right|^{2} d V} \text { and } \\
b_{n}=\frac{1}{k_{n} c} \frac{\left.\int_{V} u^{\star}(\boldsymbol{r}) \frac{\partial \psi_{2}}{\partial t}\right|_{t=0} d V}{\int_{V}\left|u_{n}(\boldsymbol{r})\right|^{2} d V} .
\end{gathered}
$$

As advertised at the beginning of this Section, we shall illustrate the use of this machinery by trying it out on a vibrating membrane. The transverse vibrations of a horizontal membrane of rectangular shape that is stretched equally with a tension $\tau$ in all directions will satisfy the two- dimensional wave equation

$$
\frac{\partial^{2} \psi}{\partial x^{2}}+\frac{\partial^{2} \psi}{\partial y^{2}}=\frac{1}{c^{2}} \frac{\partial^{2} \psi}{\partial t^{2}}, \quad c^{2}=\frac{\tau}{\mu}
$$

where $\mu$ is the mass per unit area and $\psi(x, y ; t)$ is the vertical displacement of the membrane at any point $(x, y)$ and time $t$. We shall set up our coordinate axes along two of the edges of the membrane. Then, assuming that it is fixed along all four of its edges and that it has sides of length $a$ and $b$, the boundary conditions for this problem are

$$
\begin{aligned}
& \psi(0, y ; t)=\psi(a, y ; t)=0 \\
& \psi(x, 0 ; t)=\psi(x, b ; t)=0 \quad \text { for all } t .
\end{aligned}
$$

As we have just seen, the solution can be expressed as

$$
\psi(x, y ; t)=\sum_{v}\left[a_{v} \cos k_{v} c t+b_{v} \sin k_{v} c t\right] u_{v}(x, y)
$$


with

$$
\frac{\partial^{2} u_{v}}{\partial x^{2}}+\frac{\partial^{2} u_{v}}{\partial y^{2}}+k_{v}^{2} u_{v}(x, y)=0
$$

and

$$
u_{v}(0, y)=u_{v}(a, y)=u_{v}(x, 0)=u_{v}(x, b)=0 \text {. }
$$

Setting $u_{v}(x, y)=X(x) Y(y)$ the Helmholtz equation in (10.8.15) separates into the eigenvalue equations

$$
\frac{d^{2} X}{d x^{2}}=-\lambda_{1} X(x) \text { and } \frac{d^{2} Y}{d y^{2}}=-\lambda_{2} Y(y)
$$

subject to

$$
X(0)=X(a)=0 \text { and } Y(0)=Y(b)=0,
$$

where $\lambda_{1}+\lambda_{2}=k_{v}^{2}$. These are identical to the eigenvalue equation in the stretched string problem and so we know that the eigenvalues are

$$
\lambda_{1}=\frac{m^{2} \pi^{2}}{a^{2}}, m=1,2, \ldots \text { and } \lambda_{2}=\frac{n^{2} \pi^{2}}{b^{2}}, n=1,2, \ldots
$$

and the corresponding eigenfunctions are

$$
u_{v}(x, y)=u_{m, n}(x, y)=X_{m}(x) Y_{n}(y)=\sin \frac{m \pi x}{a} \sin \frac{n \pi y}{b} .
$$

Further, since $k_{v}^{2}=\lambda_{1}+\lambda_{2}=\pi^{2}\left(\frac{m^{2}}{a^{2}}\right)$, the corresponding time dependence is given by

$$
T_{m, n}(t)=a_{m, n} \cos \omega_{m, n} t+b_{m, n} \sin \omega_{m, n} t
$$

with $\omega_{m, n}=\pi c \sqrt{\frac{m^{2}}{a^{2}}+\frac{n^{2}}{b^{2}}}, m$ and $n=1,2, \ldots$ Thus, each pair of integers $(m, n)$ defines a distinct normal mode of vibration of the membrane and the complete solution of the two-dimensional wave equation with homogeneous conditions at rectangular boundaries is the superposition

$$
\psi(x, y ; t)=\sum_{m=1}^{\infty} \sum_{n=1}^{\infty}\left[a_{m, n} \cos \omega_{m, n} t+b_{m, n} \sin \omega_{m, n} t\right] \sin \frac{m \pi x}{a} \sin \frac{n \pi y}{b} .
$$

This is a double Fourier sine series and so equations (10.8.10) and (10.8.11) for the coefficients reproduce the familiar Euler formulae. Specifically, if we impose initial conditions

$$
\psi(x, y ; 0)=u_{0}(x, y) \text { and }\left.\frac{\partial \psi}{\partial t}\right|_{t=0}=v_{0}(x, y)
$$

we have

$$
a_{m, n}=\frac{4}{a b} \int_{0}^{a} \int_{0}^{b} u_{0}(x, y) \sin m \pi \frac{x}{a} \sin \frac{n \pi y}{b} d x d y
$$


and

$$
b_{m, n}=\frac{4}{a b \omega_{m, n}} \int_{0}^{a} \int_{0}^{b} v_{0}(x, y) \sin \frac{m \pi x}{a} \sin \frac{n \pi y}{b} d x d y .
$$

It is interesting to explore the properties of the individual normal modes. Because

$$
\sin \frac{m \pi x}{a}=0 \text { at } x=\frac{a}{m}, \frac{2 a}{m}, \ldots,(m-1) \frac{a}{m}
$$

and

$$
\sin \frac{n \pi y}{b}=0 \text { at } y=\frac{b}{n}, \frac{2 b}{n}, \ldots,(n-1) \frac{b}{n},
$$

the $(m, n)$ normal mode,

$$
\psi_{m, n}(x, y ; t)=\sin \frac{m \pi x}{a} \sin \frac{n \pi y}{b}\left[a_{m, n} \cos \omega_{m, n} t+b_{m, n} \sin \omega_{m, n} t\right],
$$

has $(m-1)$ nodal lines parallel to the y-axis and $(n-1)$ nodal lines parallel to the $\mathrm{x}$-axis. Every point on each of these lines remains at rest for all $t$.

Two modes can possess the same frequency if $\frac{a}{b}$ is a rational number. When that happens we say that the frequency is degenerate because it is associated with more than one eigenfunction. A simple example is afforded by a square membrane since then every pair of transposed integers defines a pair of normal modes with the same frequency. For instance, the $(2,1)$ and $(1,2)$ modes both have frequency $\frac{\sqrt{5} \pi c}{a}$. Moreover, any linear combination of the $(2,1)$ and $(1,2)$ modes,

$$
\psi(x, y ; t)=\left(A \sin \frac{2 \pi x}{a} \sin \frac{\pi y}{a}+B \sin \frac{\pi x}{a} \sin \frac{2 \pi y}{a}\right) \cos \frac{\sqrt{5} c \pi}{a} t,
$$

represents a harmonic motion with the same frequency. These solutions are called hybrid modes and are vectors in the space spanned by the normal modes. The hybrid modes have nodal curves whose location depends on the relative value of the coefficients $A$ and $B$. 